\title{
A Generalized Multiconductor Transmission Line Model and Optimized Method for the Solution of the MTL Equations
}

\author{
Chaoqun Jiao, ${ }^{1,2}$ Zhanjun Xia, ${ }^{1,2}$ and W. N. Fu ${ }^{3}$ \\ ${ }^{1}$ School of Electrical Engineering, Beijing Jiaotong University, No. 3 Shang Yuan Cun, Haidian District, Beijing 100044, China \\ ${ }^{2}$ State Key Laboratory of Millimeter Waves, Nanjing 210096, China \\ ${ }^{3}$ Department of Electrical Engineering, The Hong Kong Polytechnic University, Hung Hom, Kowloon, Hong Kong
}

Correspondence should be addressed to Chaoqun Jiao, chaoqunjiao@126.com

Received 13 December 2011; Accepted 2 February 2012

Academic Editor: Mandeep Jit Singh

Copyright (C 2012 Chaoqun Jiao et al. This is an open access article distributed under the Creative Commons Attribution License, which permits unrestricted use, distribution, and reproduction in any medium, provided the original work is properly cited.

\begin{abstract}
A generalized multiconductor transmission line (MTL) model is developed for modeling of wide frequency transient response on busbars, cables and core-type transformer windings. Different from the traditional MTL model, the equations of the generalized MTL model are built in the cylindrical coordinate system beside rectangular coordinate system. Based on further discussion, it is found that generalized MTL model could be changed to MTL model where all lines have the same length as to the core-type transformer windings. Then, the optimized solution based on Time domain finite element method (TDFEM) is developed for the above MTL equations. It avoids numerical oscillation of the finite difference time domain (FDTD) method. The numerical results are in agreement with ones calculated by Bergeron's method and FDTD method.
\end{abstract}

\section{Introduction}

Very fast transient overvoltages (VFTOs) generated by switching operation of circuit breakers and disconnected switches could cause a voltage oscillation on the busbars, cables, and the windings inside the transformer connected. Because of the large dimension of power substations, the FDTD method and the method of moment (MOM) are not convenient to be used. Lumped circuit models have been used to analyze the transient response. But for the analysis involving the VFTO of much higher frequencies, the MTL theory is applied and a hybrid method of MTLs model and antenna theory is proposed. The transient wave process along the transmission line is calculated first, and then the field is calculated [1-6].

In order to analyze the wave processes, time-domain method is always recommended. Bergeron's method is a timedomain method, which has been widely used to calculate the wave processes in power system and has been implemented in EMTP code $[7,8]$. However, only the voltage and current at some specified nodes can be calculated by this method. It is not effective for the calculation of the complete wave processes of voltage and current distributed along the MTLs. Nonetheless, the problem can be solved by FDTD [9]. Paul proposed an iterative algorithm for the lossy MTLs with arbitrary loads [10]. Lu et al. improved and complemented Paul's method [11-14]. Because of the Gibbs effect of FDTD method, Park and Lei develop the finite-element method (FEM) and TDFEM for this problem $[15,16]$.

\section{Generalized MTLs Model}

The typical models of the busbars, cables, core-type transformer windings, and the print circuit board with $n+1$ conductors and the MTLs model of them are shown in Figure 1. We suppose that the propagation of the MTLs is along $z$-direction and its per-unit-length inductance $\mathbf{L}$, capacitance $\mathbf{C}$, conductance $\mathbf{G}$, and resistance $\mathbf{R}$ matrixes have been known, which can be determined by the geometry of the MTLs and its surrounding media. The governing equations, called telegraph equations, can be expressed as follows:

$$
\begin{gathered}
\frac{\partial \mathbf{U}(z, t)}{\partial z}+\mathbf{L} \frac{\partial \mathbf{I}(z, t)}{\partial t}+\mathbf{R}(z, t)=\mathbf{V}_{F} \\
\frac{\partial \mathbf{I}(z, t)}{\partial z}+\mathbf{C} \frac{\partial \mathbf{U}(z, t)}{\partial t}+\mathbf{G U}(z, t)=\mathbf{I}_{F}
\end{gathered}
$$




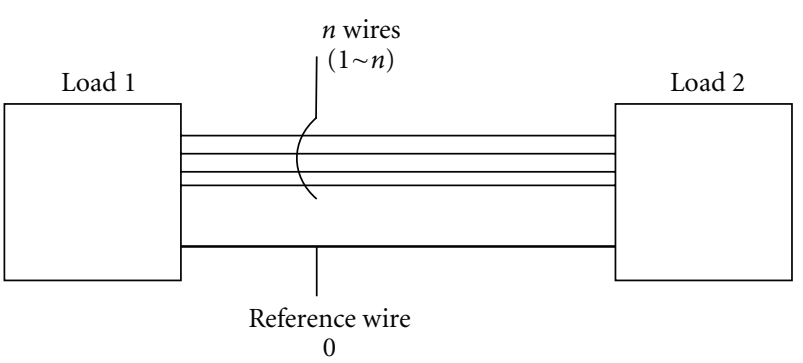

(a)

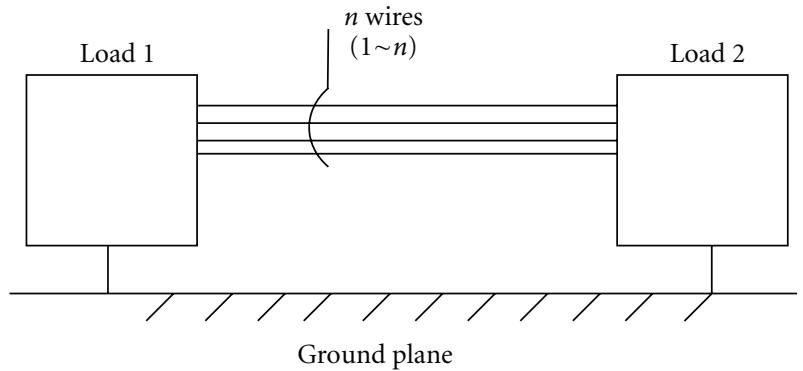

(b)

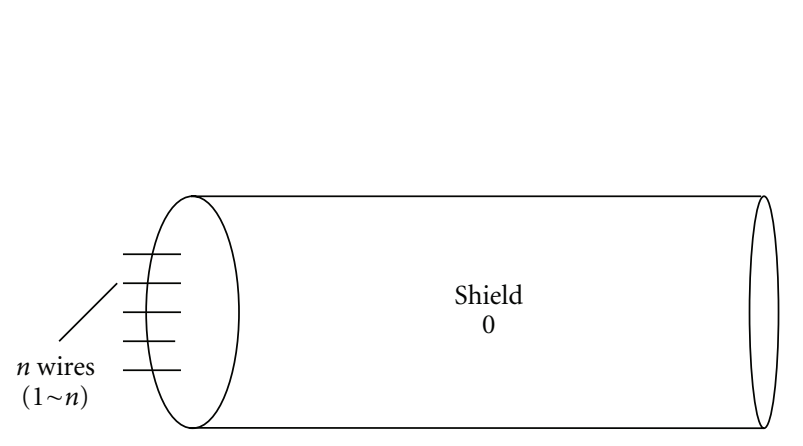

(c)

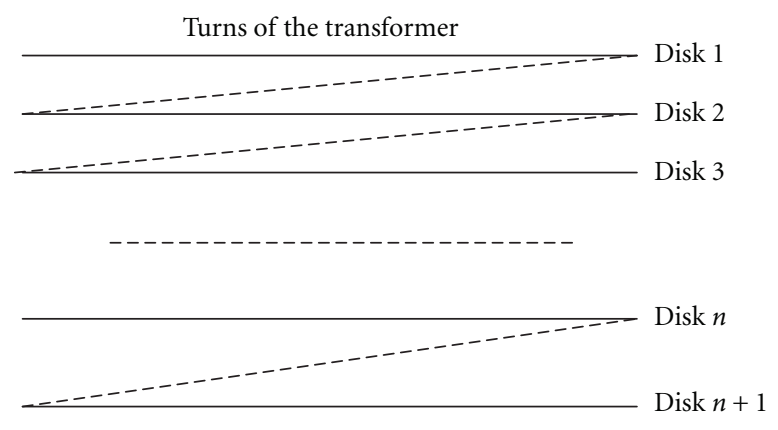

(d)

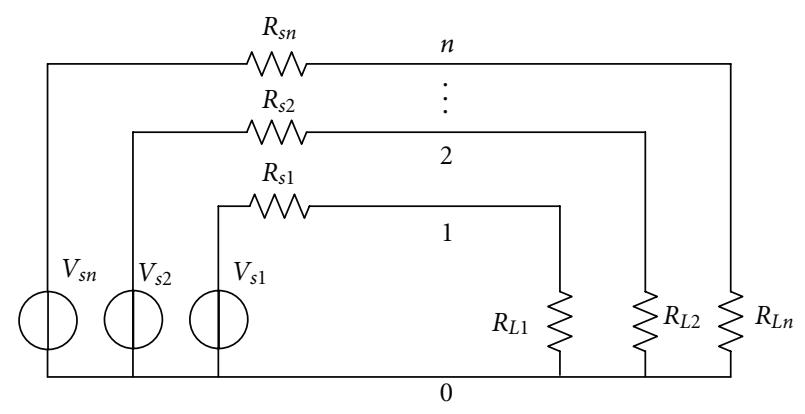

(e)

FIgURE 1: Examples and computational model of MTLs.

where $z$ is the direction of wave propagation; $\mathbf{U}$ and $\mathbf{I}$ denote the voltage and current vectors on the $z$ point of line at $t$ moment, respectively; $\mathbf{V}_{F}$ and $\mathbf{I}_{F}$, respectively, denotes the excitation voltage source and motivation current source vectors on the $z$ point at time $t$.

The turn-to-turn MTL model is usually used for VFTO distribution analysis of transformer windings. The MTL model requires all lines to have the same length. However, in large power transformer the length difference between turns could be large. When all turn lengths are assumed to be the same, the coefficient matrices of the MTL equation are not real unit length parameters. Also, it is hard to explain if the same length assumption could cause inaccuracy in MTL model. In [17] the circular MTL (CMTL) equations for the winding are developed, and the characteristics of the coefficient matrices in the formulas are analyzed. The derivation of the circular MTL equations for core-type transformer windings is similar to that of the traditional MTL equations for busbars and cables. The propagation direction of the wave is the same as the winding direction; that is, the wave propagates along direction $\varphi$ :

$$
\begin{gathered}
\frac{\partial \mathbf{U}}{\partial \varphi}+\mathbf{L}_{\varphi} \frac{\partial \mathbf{I}}{\partial t}+\mathbf{R}_{\varphi} \mathbf{I}=0, \\
\frac{\partial \mathbf{I}}{\partial \varphi}+\mathbf{C}_{\varphi} \frac{\partial \mathbf{U}}{\partial t}+\mathbf{G}_{\varphi} \mathbf{U}=0 .
\end{gathered}
$$

$\mathbf{L}_{\varphi}$ and $\mathbf{C}_{\varphi}$ are the per-unit-radian inductance and capacitance matrix, respectively. They can be calculated by dividing the lump inductance and capacitance matrix by $2 \pi$. Based on the circular MTL equations, the rationality of using equal-length assumption in the simulation of actual winding can be discussed. Letting $r_{0}$ be an assumed radius and not 


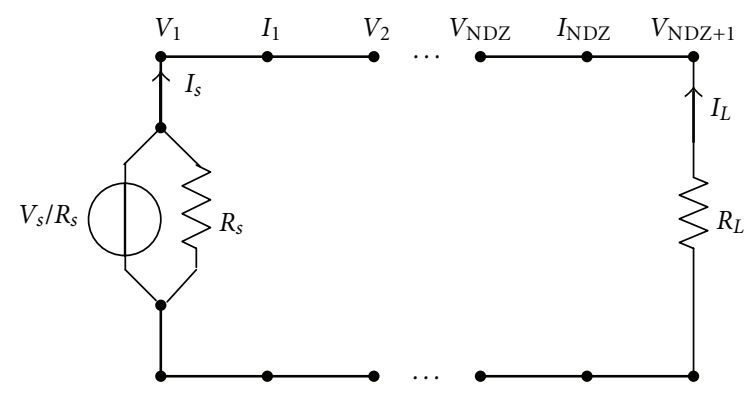

FIgUre 2: The MTLs elements in space and time.

considering lossless transmission lines, take place of $\varphi$ in (2) with $z=r_{0} \varphi$, then there is

$$
\begin{aligned}
& \frac{\partial \mathbf{U}}{\partial \varphi}+\mathbf{L}_{z} \frac{\partial \mathbf{I}}{\partial t}=0 \\
& \frac{\partial \mathbf{I}}{\partial \varphi}+\mathbf{C}_{z} \frac{\partial \mathbf{U}}{\partial t}=0 .
\end{aligned}
$$

Assume that $\mathbf{L}$ and $\mathbf{C}$ are the lumped inductance and capacitance matrixes; respectively, there is

$$
\begin{aligned}
\mathbf{L}_{z} & =\frac{1}{r_{0}} \mathbf{L}_{\varphi}=\frac{1}{2 \pi r_{0}} \mathbf{L}, \\
\mathbf{C}_{z} & =\frac{1}{r_{0}} \mathbf{C}_{\varphi}=\frac{1}{2 \pi r_{0}} \mathbf{C} .
\end{aligned}
$$

If $r_{0}$ is the average radius of the turns [17], then $2 \pi r_{0}$ would be the average length of the turns. In this condition, (3) represents a MTL model where all lines have the same length $2 \pi r_{0}$, and they have the same solutions as (2). So CMTL model could be changed to MTL model where all lines have the same length, with the prerequisite that the coefficient matrixes are calculated correctly in the original circular system.

So the generalized MTL model is (2). They can be changed to MTL model where all lines have the same length.

\section{Optimized Method for Solutions of the MTL Equations}

The authors in [15] describe a false time-domain finite-element method for lossy transmission time analysis. The two nodes of a fist-order finite element are placed in such away that each voltage element contains an interpolation node for the current and each current element contains an interpolation node for the voltage as Figure 2. The final equations are

$$
\begin{aligned}
\mathbf{I}_{k}^{n+1 / 2}= & \frac{2 L-\mathbf{R} \Delta t}{2 L+\mathbf{R} \Delta t} \mathbf{I}_{k}^{n-1 / 2} \\
& -\frac{2 \Delta t}{(2 L+\mathbf{R} \Delta t) \Delta z}\left(\mathbf{V}_{k+1}^{n}-\mathbf{V}_{k}^{n}\right), \\
\mathbf{V}_{k}^{n+1}= & \frac{2 C-\mathbf{G} \Delta t}{2 C+\mathbf{G} \Delta t} \mathbf{V}_{k}^{n} \\
& -\frac{2 \Delta t}{(2 C+\mathbf{G} \Delta t) \Delta z}\left(\mathbf{I}_{k+1}^{n+1 / 2}-\mathbf{I}_{k}^{n+1 / 2}\right) .
\end{aligned}
$$

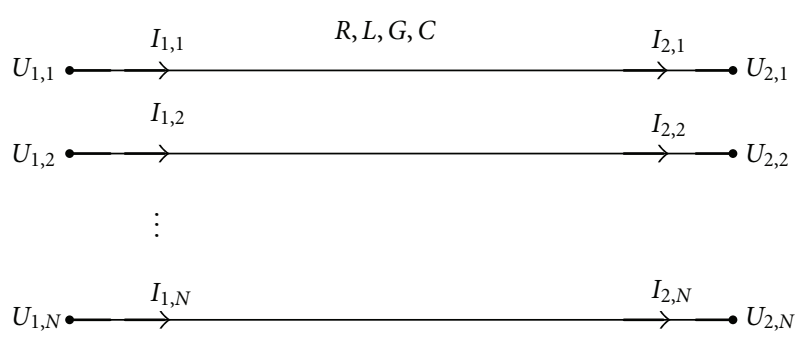

FIgURe 3: MTLs' finite element.

They can be obtained by multiplying and dividing both sides of (6) and (7) in [10], respectively, by $2 \Delta t$ and $\Delta z$ and then not considering $\mathbf{V}_{F}$.

$$
\begin{aligned}
\left(\mathbf{L} \frac{\Delta z}{\Delta t}+\frac{\mathbf{R}}{2} \Delta z\right) \mathbf{I}_{k}^{n+1 / 2}= & \left(\mathbf{L} \frac{\Delta z}{\Delta t}-\frac{\mathbf{R}}{2} \Delta z\right) \mathbf{I}_{k}^{n-1 / 2} \\
& -\left(\mathbf{V}_{k+1}^{n}-\mathbf{V}_{k}^{n}\right)+\frac{\Delta z}{2}\left(\mathbf{V}_{F k}^{n+1 / 2}+\mathbf{V}_{F k}^{n-1 / 2}\right) \\
\left(\mathbf{C} \frac{\Delta z}{\Delta t}+\frac{\mathbf{G}}{2} \Delta z\right) \mathbf{V}_{k}^{n+1}= & \left(\mathbf{C} \frac{\Delta z}{\Delta t}-\frac{\mathbf{G}}{2} \Delta z\right) \mathbf{V}_{k}^{n} \\
& -\left(\mathbf{I}_{k}^{n+1 / 2}-\mathbf{I}_{k-1}^{n+1 / 2}\right)+\frac{\Delta z}{2}\left(\mathbf{I}_{F k}^{n+1}+\mathbf{I}_{F k}^{n}\right)
\end{aligned}
$$

Following [16], the TDFEM to analyze single transmission is extended to MTLs. When solving Telegrapher's equation by the finite-element method, the MTLs are divided into finite elements. Figure 3 shows the finite-element of such MTLs.

The starting point to obtain a local matrix and vector of line finite element is the Telegrapher's equations:

$$
\begin{gathered}
\frac{\partial \mathbf{U}(z, t)}{\partial z}+\mathbf{L} \frac{\partial \mathbf{I}(z, t)}{\partial t}+\mathbf{R I}(z, t)=0, \\
\frac{\partial \mathbf{I}(z, t)}{\partial z}+\mathbf{C} \frac{\partial \mathbf{U}(z, t)}{\partial t}+\mathbf{G} \mathbf{U}(z, t)=0 .
\end{gathered}
$$

Using weighted residual method, a system of two residual equations is obtained as follows:

$$
\begin{aligned}
& \int_{z_{1}}^{z_{2}}\left[\frac{\partial \mathbf{U}(z, t)}{\partial z}+\mathbf{L} \frac{\partial \mathbf{I}(z, t)}{\partial t}+\mathbf{R I}(z, t)\right] W_{1} d z=0 \\
& \int_{z_{1}}^{z_{2}}\left[\frac{\partial \mathbf{I}(z, t)}{\partial z}+\mathbf{C} \frac{\partial \mathbf{U}(z, t)}{\partial t}+\mathbf{G V}(z, t)\right] W_{1} d z=0 .
\end{aligned}
$$

Choosing the weighting functions $W_{1}=1$, voltage and current waves over the finite element can be approximated by a linear combination of interpolation functions such as

$$
\begin{aligned}
& \mathbf{U}(z, t)=\sum_{k=1}^{2} \Phi_{k}(z) \mathbf{U}_{k}(t), \\
& \mathbf{I}(z, t)=\sum_{k=1}^{2} \Phi_{k}(z) \mathbf{I}_{k}(t) .
\end{aligned}
$$




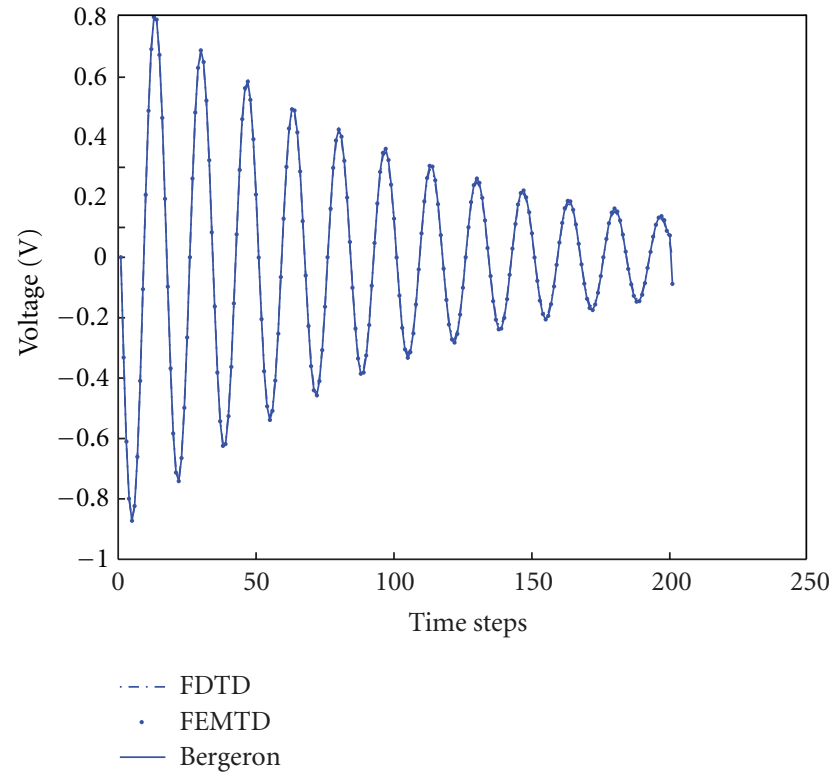

(a)

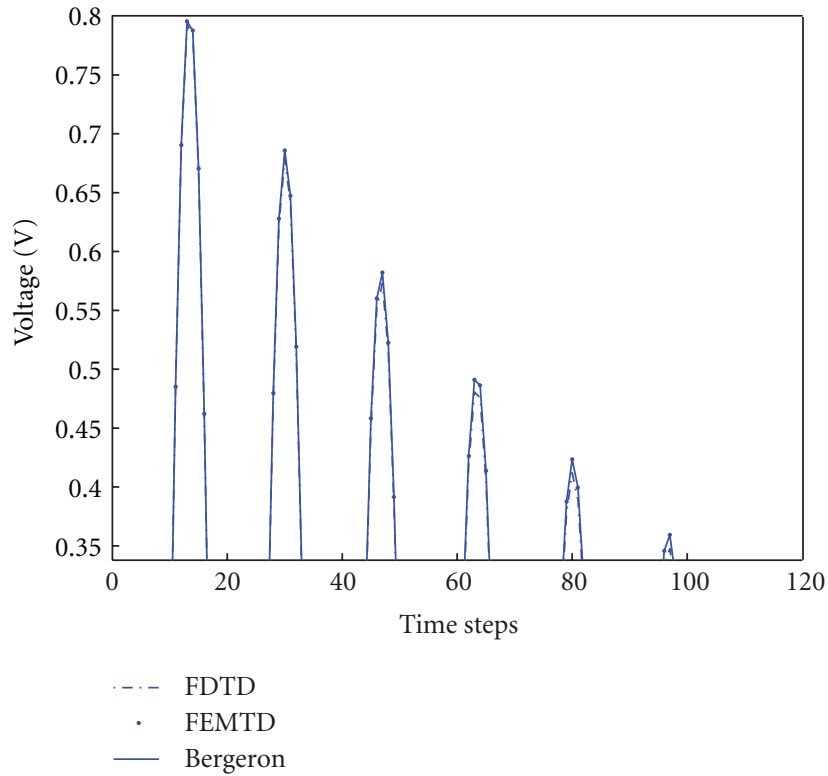

(b)

FIGURE 4: Voltage waveform on a distortionless line.

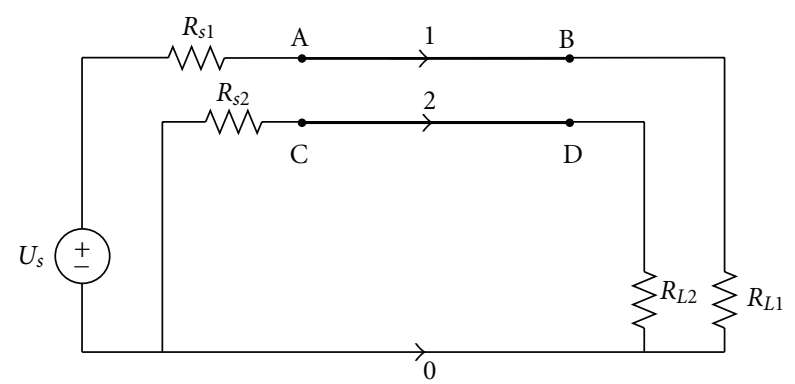

FIgURE 5: Crosstalk problem of two wires.

Variables $\mathbf{U}_{k}(t)$ and $\mathbf{I}_{k}(t)$ represent voltage and current waves associated to node " $k$ ", $k=1,2$ of the finite element:

$$
\begin{aligned}
\mathbf{U}_{k}(t) & =\left[U_{k, 1}(t), U_{k, 2}(t), \ldots, U_{k, N}(t)\right]^{T}, \\
\mathbf{I}_{k}(t) & =\left[I_{k, 1}(t), I_{k, 2}(t), \ldots, I_{k, N}(t)\right]^{T},
\end{aligned}
$$

where $\Phi_{k}(z), k=1,2$ represent interpolation functions, and they are

$$
\begin{aligned}
& \Phi_{1}(z)=\operatorname{diag}\left[\frac{z_{2}-z}{z_{2}-z_{1}}, \frac{z_{2}-z}{z_{2}-z_{1}}, \ldots, \frac{z_{2}-z}{z_{2}-z_{1}}\right], \\
& \Phi_{2}(z)=\operatorname{diag}\left[\frac{z-z_{1}}{z_{2}-z_{1}}, \frac{z-z_{1}}{z_{2}-z_{1}}, \ldots, \frac{z-z_{1}}{z_{2}-z_{1}}\right] .
\end{aligned}
$$

Time integration performed on the system of ordinary differential equations is carried out using explicit/implicit mixed procedure. Using the Newton-Raphson method in the case of nonlinear distributed parameters, local system of algebraic equations is

$$
\begin{aligned}
& {\left[\begin{array}{cc}
-\theta \Delta t & -\theta \Delta t \\
-\frac{\Delta z}{2}(C+\theta \Delta t G) & -\frac{\Delta z}{2}(C+\theta \Delta t G)
\end{array}\right]\left[\begin{array}{l}
\Delta U_{1} \\
\Delta U_{2}
\end{array}\right]} \\
& +\left[\begin{array}{cc}
-\frac{\Delta z}{2}(L+\theta \Delta t R) & -\frac{\Delta z}{2}(L+\theta \Delta t R) \\
-\theta \Delta t & -\theta \Delta t
\end{array}\right]\left[\begin{array}{l}
\Delta I_{1} \\
\Delta I_{2}
\end{array}\right] \\
& =\left[\begin{array}{c}
-\Psi_{1} \\
-\Psi_{2}
\end{array}\right], \\
& \Psi_{1}=\mathbf{R} \frac{\Delta z}{2} \Delta t\left[\mathbf{I}_{1}^{n}+\mathbf{I}_{2}^{n}\right]+\Delta t\left[\mathbf{U}_{2}^{n}-\mathbf{U}_{1}^{n}\right], \\
& \Psi_{2}=\mathbf{G} \frac{\Delta z}{2} \Delta t\left[\mathbf{U}_{1}^{n}+\mathbf{U}_{2}^{n}\right]+\Delta t\left[\mathbf{I}_{2}^{n}-\mathbf{I}_{1}^{n}\right],
\end{aligned}
$$

where $\Delta U_{k}=U_{k}^{n+1}-U_{k}^{n}, \Delta I_{k}=I_{k}^{n+1}-I_{k}^{n}, k=1,2, \Delta t=$ $\operatorname{diag}\{\Delta t, \Delta t, \ldots, \Delta t\}, \Delta t$ is the time step, and $\Delta z=z_{1}-z_{2}$.

Assembly of global system of equations is based on satisfaction of continuity equation (Kirchhoff's current law) for each node in electrical network. Therefore, it is necessary to transform system (13) into

$$
\left[\begin{array}{l}
\Delta I_{1} \\
\Delta I_{2}
\end{array}\right]=-\mathbf{A}\left[\begin{array}{l}
\Delta U_{1} \\
\Delta U_{2}
\end{array}\right]+\mathbf{B}\left[\begin{array}{l}
-\Psi_{1} \\
-\Psi_{2}
\end{array}\right],
$$

where $\mathbf{A}$ and $\mathbf{B}$ matrices can be obtained by simple numerical procedure.

Previously defined local system of various finite elements has to be assembled. To obtain global system, it is necessary to satisfy continuity equation in each node of electric circuit. 


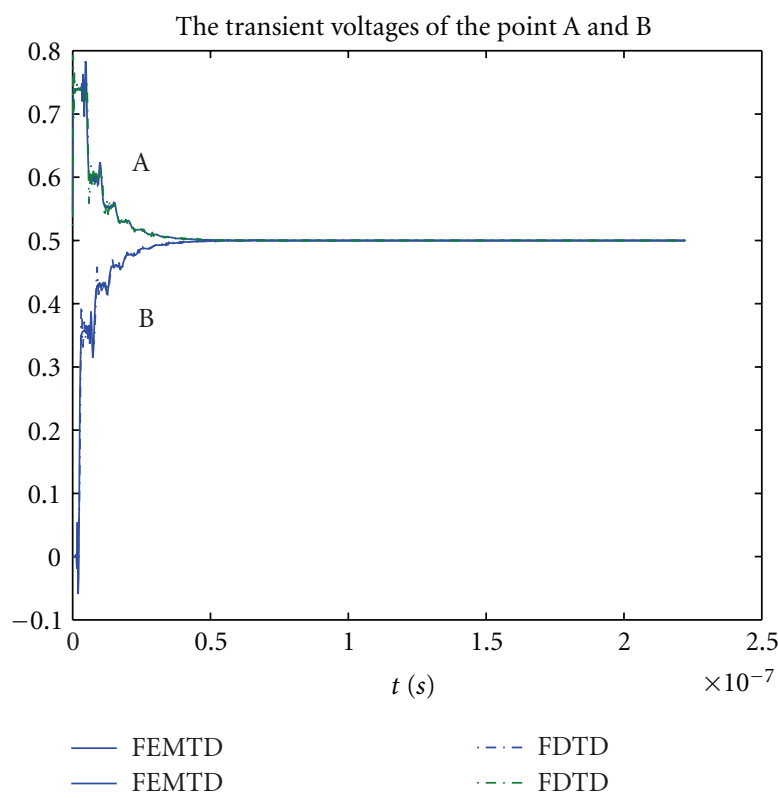

(a)

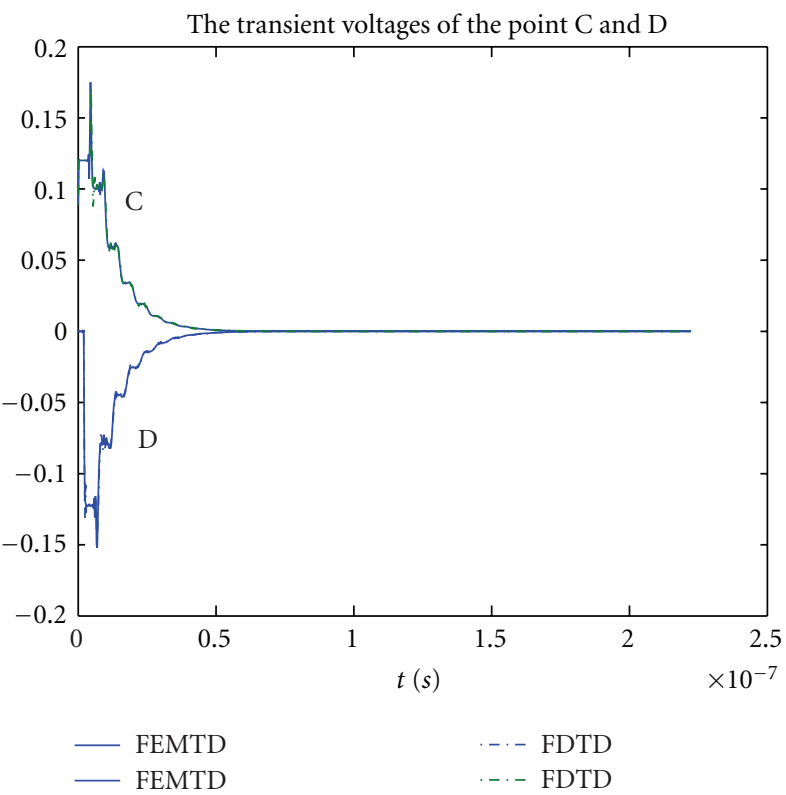

(c)

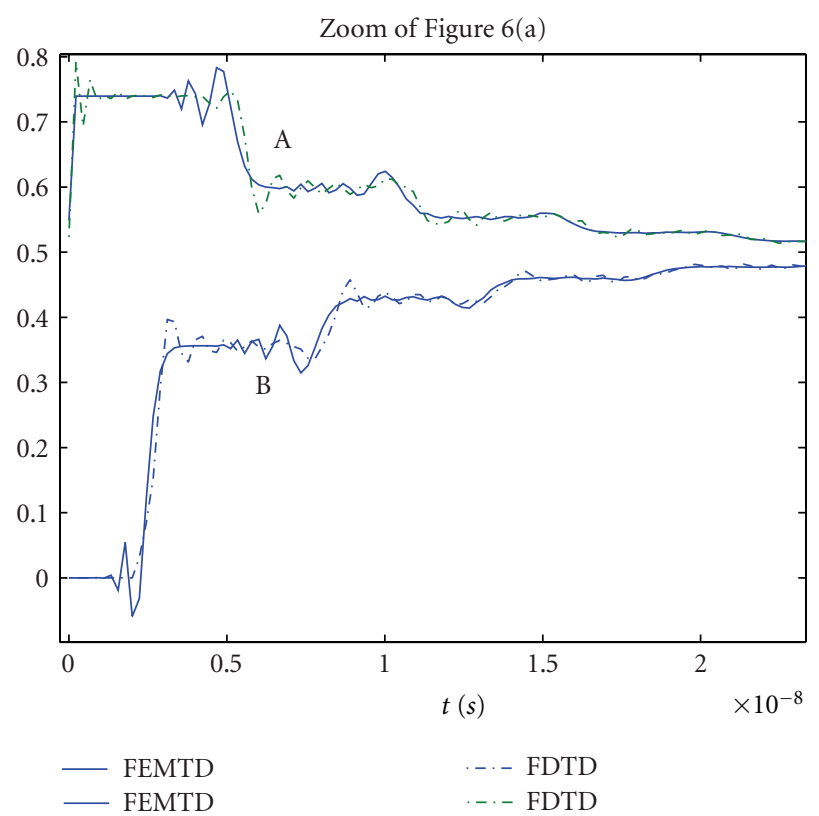

(b)

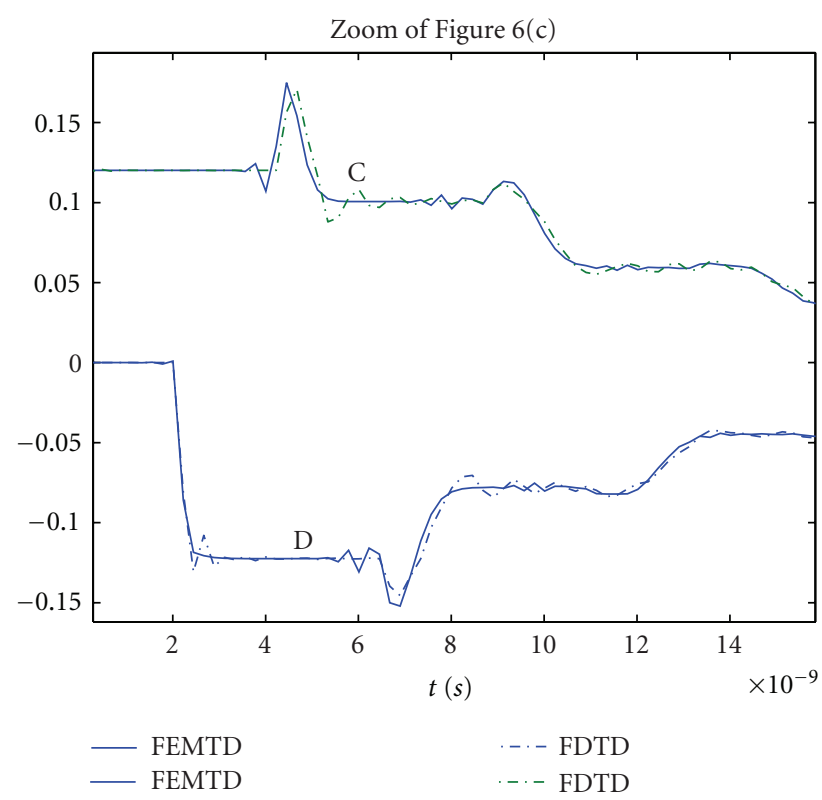

(d)

FIgURE 6: Comparison of the results of TDFEM and FDTD method.

Each node has its local and global index. For node with global index " $s$," one can write integral form of continuity equation as

$$
\begin{array}{r}
\int_{t_{1}}^{t_{2}}\left[\sum_{j=1}^{m}(-1)^{p}\left(I_{j, p}\right)_{s}+\left(I_{F}\right)_{s}\right] d t=0 \\
s=1, M ; \quad p=1 \text { or } 2 .
\end{array}
$$

The matrix relation (15) for each finite element is

$$
\begin{aligned}
& \Delta I_{1}=-A_{11} \Delta U_{1}-A_{12} \Delta U_{2}-B_{11} \Psi_{1}-B_{12} \Psi_{2}, \\
& \Delta I_{2}=-A_{21} \Delta U_{1}-A_{22} \Delta U_{2}-B_{21} \Psi_{1}-B_{22} \Psi_{2}
\end{aligned}
$$


for the case when the local node is " 1 " is

$$
\begin{aligned}
\theta\left[\begin{array}{ll}
A_{11} & A_{12}
\end{array}\right]\left[\begin{array}{c}
\Delta U_{1} \\
\Delta U_{2}
\end{array}\right]= & \theta\left(-B_{11} \Psi_{1}-B_{12} \Psi_{2}\right) \\
& +\left[(1-\theta) I_{1}^{n}+\theta I_{1}^{n+1}\right] \\
& +\left[(1-\theta) I_{F}^{n}+\theta I_{F}^{n+1}\right]
\end{aligned}
$$

for the case when the local node is " 2 " is

$$
\begin{aligned}
\theta\left[\begin{array}{ll}
A_{21} & A_{22}
\end{array}\right]\left[\begin{array}{l}
\Delta U_{1} \\
\Delta U_{2}
\end{array}\right]= & \theta\left(-B_{21} \Psi_{1}-B_{22} \Psi_{2}\right) \\
& +\left[(1-\theta) I_{2}^{n}+\theta I_{2}^{n+1}\right] \\
& +\left[(1-\theta) I_{F}^{n}+\theta I_{F}^{n+1}\right] .
\end{aligned}
$$

Summing up the contributions of all incident finite elements over all nodes, global system for $k$-iterative step becomes

$$
\mathbf{A}_{g}\left[\Delta \mathbf{U}_{g}\right]=\mathbf{b}_{g}
$$

The present numerical procedure is stable for $0.5 \leq \theta \leq 1$.

The first example is the example in [15]. Signals on a distortionless lossy line experience attenuation without any distortion. A line with $\mathbf{C}=l \mathrm{~F} / \mathrm{m}, \mathbf{L}=1 \mathrm{H} / \mathrm{m}, \mathbf{R}=1 \Omega$, and $\mathbf{G}=10 \mathrm{~S} / \mathrm{m}$ is excited by a sinusoidal source with frequency of $60 \mathrm{~Hz}$ and amplitude of $1 \mathrm{~V}$. The resulting waveforms of the TDFEM, the FDTD method, and Bergeron's method and the zoomed waveforms of them are shown in Figure 4.

The other example is the crosstalk problem of two wires as shown in Figure 5. The voltage source is step function and its magnitude is $1 \mathrm{~V} . R_{s 1}=50, R_{s 2}=50, R_{L 1}=50, R_{s 2}=50$. The length of the MTLs is $0.5 \mathrm{~m} . \Delta z=0.005 \mathrm{~m}$. The per-unitlength parameters are the same as in [9]. The comparison of the results of the TDFEM and FDTD method is shown in Figure 6.

From Figures 4 and 6, we can see that the results of the TDFEM basically agree with the results of FDTD method and Bergeron's method in the sinusoidal steady analysis and transient analysis. But from the zoom part in the Figure 6, in transient analysis, the results of the FDTD have numerical oscillation. As to Bergeron's method, only the voltage and current at some specified nodes can be calculated. And the TDFEM can overcome these disadvantages. So the TDFEM would be the optimized method for analyzing the traditional MTLs. But the FDTEM is complicated; so, for the overhead lines with multi-layer soil, the frequency-dependent overhead lines nonuniform MTL, and the FDTD is as the first point for its simplicity [10-12].

\section{Conclusion}

In this paper, a generalized MTL model is developed for modeling of wide frequency transient response on busbars, cables and core-type transformer windings. Different from the traditional MTL model, the equations of the generalized
MTL model are built in the cylindrical coordinate system. Then, the optimized solution based on TDFEM is developed for the above MTL equations. In the sinusoidal steady analysis, its results agree with ones of the FDTD method and Bergeron's method. But in transient analysis, it avoids of numerical oscillation of the FDTD method. Comparing with Bergeron's method, all voltages and currents along the MTLs can be calculated with the TDFEM.

\section{Acknowledgments}

This work was supported by the National Natural Science Fund (nos. 50807003 and 51107003), the State Key Laboratory of Millimeter Waves (no. K201012), and the Research Fund of the Fundamental Research Funds for the Central Universities of Beijing Jiaotong University (no. 2011JBM110).

\section{References}

[1] C. M. Wiggins and S. E. Wright, "Switching transient fields in substations," IEEE Transactions on Power Delivery, vol. 6, no. 2, pp. 591-600, 1991.

[2] Z. Wang, "Model for transient analysis in large transformer windings," Journal of Tsinghua University, vol. 33, no. 1, pp. 25-32, 1993 (Chinese).

[3] M. Popov, L. Van Der Sluis, G. C. Paap, and H. De Herdt, "Computation of very fast transient overvoltages in transformer windings," IEEE Transactions on Power Delivery, vol. 18, no. 4, pp. 1268-1274, 2003.

[4] F. E. Gardiol, Lossy Transmission Lines, ArtechHouse, Boston, Mass, USA, 1987.

[5] P. Grivet, The Physics of Transmission Lines at High and Very High Frequencies, Academic Press, New York, NY, USA, 1970.

[6] C. M. Wiggins and S. E. Wright, "Switching transient fields in substations," IEEE Transactions on Power Delivery, vol. 6, no. 2, pp. 591-600, 1991.

[7] H. W. Dommel, "Digital computer solution of electromagnetic transients in single-and multiphase networks," IEEE Transactions on Power Apparatus and Systems, vol. 88, no. 2, pp. 734-741, 1969.

[8] H. W. Dommel and I. I. Dommel, “Transients Program, User's Manual," 1982.

[9] F. M. Tesche, M. V. Ianoz, and T. Karlsson, EMC Analysis Methods and Computation Models, John Wiley \& Sons Press, New York, NY, USA, 1996.

[10] C. R. Paul, Analysis of Multicodutor Transmission Lines, John Wiley \& Sons, New York, NY, USA, 1994.

[11] T. Lu, X. Cui, and N. Li, "Transient analysis of aerial multiconductor transmission lines with branch," IEEE Transactions on Magnetics, vol. 37, no. 5 I, pp. 3298-3302, 2001.

[12] L. Qi, T. B. Lu, and X. Cui, "Transient analysis for nonuniform multiconductor transmission lines based on the FDTD method," Proceedings of the Chinese Society of Electrical Engineering, vol. 23, no. 7, pp. 102-106, 2003.

[13] H. M. J. De Silva, A. M. Gole, J. E. Nordstrom, and L. M. Wedepohl, "Robust passivity enforcement scheme for timedomain simulation of multi-conductor transmission lines and cables," IEEE Transactions on Power Delivery, vol. 25, no. 2, pp. 930-938, 2010. 
[14] K. Noguchi, H. Rajagopalan, and Y. Rahmat-Samii, "Design of E-shaped patch antennas by using the multi-conductor transmission line mode theory," in Proceedings of Asia-Pacific Microwave Conference, pp. 84-87, 2010.

[15] S. Y. Lee, A. Konrad, and R. Saldanha, "Lossy transmission line transient analysis by the finite element method," IEEE Transactions on Magnetics, vol. 29, no. 2, pp. 1730-1732, 1993.

[16] R. Lucic, V. Jovic, and M. Kurtovic, "Simulation of electromagnetic transients on single transmission lines via the finite element method," in Proceedings of the International Conference and Exhibition on Electromagnetic Compability (EMC York '99), pp. 41-46, July 1999.

[17] Y. Yang and Z. J. Wang, "A circular multi-conductor transmission line model for simulation of very fast transient in circular windings," in Proceedings of the Progress In Electromagnetics Research Symposium, Beijing, China, 2009. 

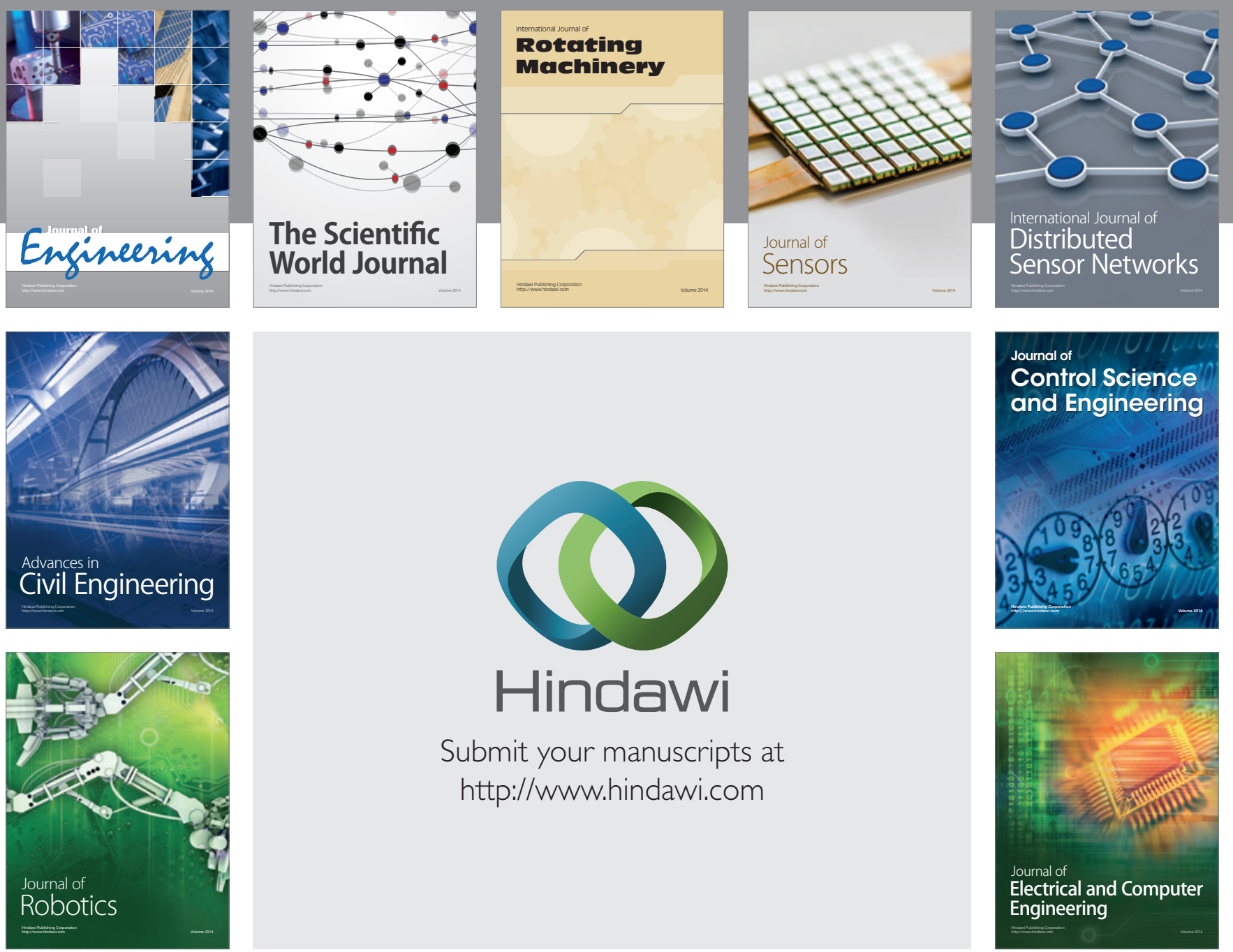

Submit your manuscripts at

http://www.hindawi.com
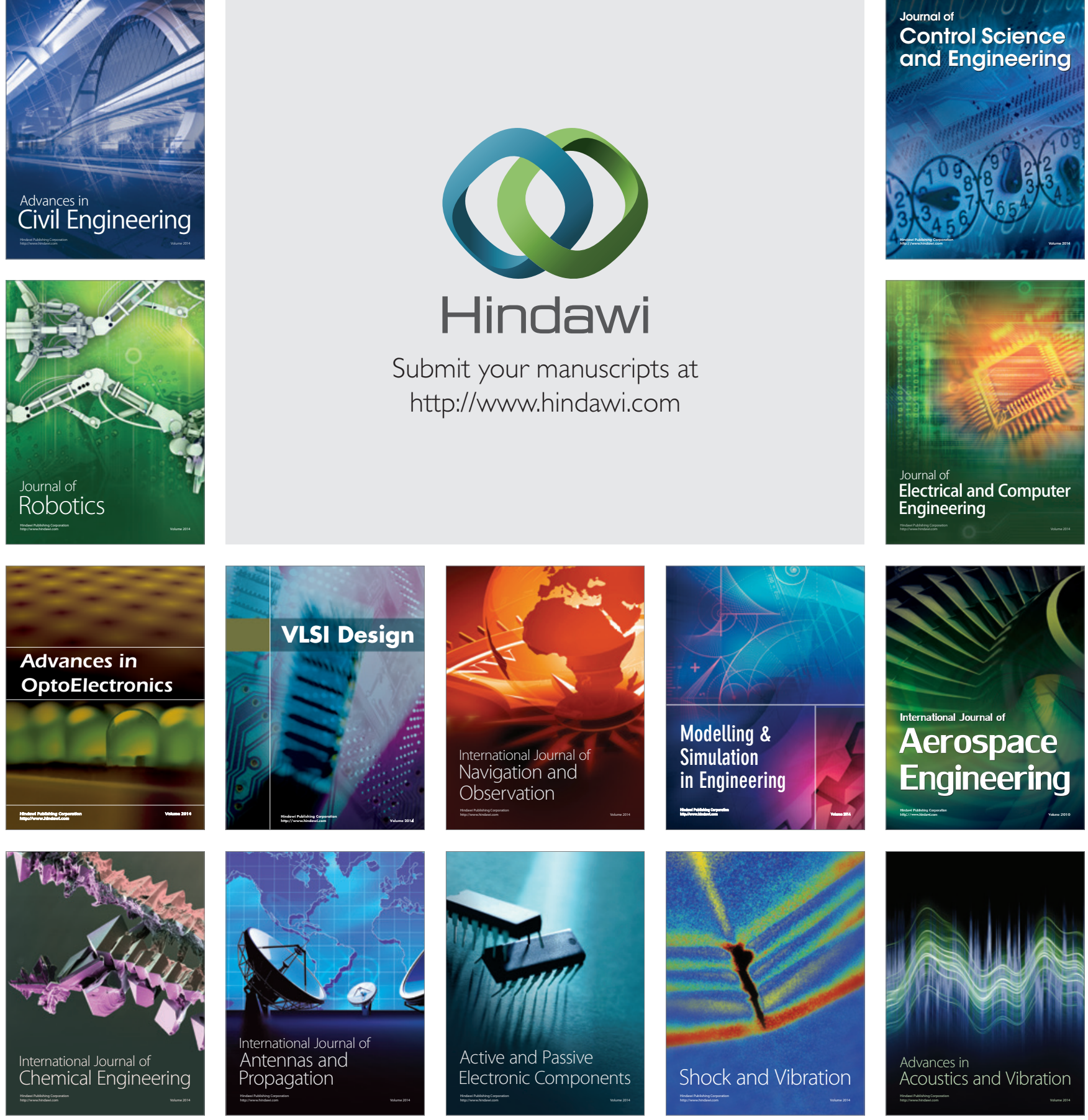